\title{
Systems Biology Strategy for Drug Potentiating through Drug Repurposing: Bisphosphonates beyond Osteoporosis Treatment
}

\section{Alexey Goltsov ${ }^{1 *}$, Zuzanna Kowalczyk ${ }^{1}$ and Rao VL Papineni ${ }^{2,3,4 *}$}

${ }^{1}$ School of Science, Engineering and Technology, Abertay University, Dundee, UK

2University of Kansas Medical Centre, KUMC, USA

${ }^{3}$ Precision X-Ray Inc. North Branford, USA

${ }^{4}$ PACT \& Health LLC. Branford, USA

\section{Editorial}

Drug repurposing (repositioning) now is an emerging direction in pharmaceutical science and drug development which aims at searching new targets for the approved drugs for treatment of completely different diseases beyond their initial registration. Among drugs which showed their effectiveness in treatment diseases besides their direct targeted diseases are such drugs as Rapamycin, immunosuppressive drug, reveals itself as promising anticancer drug [1]; metformin being the medication for the treatment of type 2 diabetes successfully showed promising as anticancer drugs [2]. Today number of drugs successfully repurposed steadily grows both in cross disease repurposing and within one class of diseases [3].

Current development strategy in drug repurposing changes from contingent and sporadic discovery of drug candidates for repurposing to a rational strategy that directly focuses on identification of novel therapeutics properties and potential targets of the established therapy and their combinations. This new strategy is accomplished by the development of the novel experimental and computational methods and tools which support and accelerate process of drug repurposing $[3,4]$. Adaptation of high throughput technologies such as nextgeneration sequencing and microarray techniques to drug repurposing aims at the screening of acting drugs and their combinations to discover off-target effects and sensitivity profiles of a drug under repurposing [5]. Computational bioinformatics methods which facilitate drug repurposing are developed based on the biostatistical data analysis oflarge gene expression repositories and interactom databases such as diseasesgenes, disease-drug, drugs-genes, and diseases-drugs-genes databases $[3,4]$. The developments of these up-to-date strategies are supported by the government's initiatives to speed up drug repositioning. For example, in the USA, the National Centre for Advancing Translational Sciences (NCATS), and National Cancer Institute (NCI) Radiation Branch (RRP), has launched the Discovering New Therapeutic Uses for Existing Molecules Programme, the Developmental Pathway Funding Scheme of the Medical Research Council (MRC) in the UK funds the projects on repurposing clinical studies, and the Netherlands Organisation for Health Research and Development has funded a project on the stimulation of drug rediscovery related to drug repositioning [4].

Beyond obvious financial benefits of drug repurposing, this tendency partly stemmed from the recent discoveries in pharmaceutical science and shift of a paradigm in drug discovery strategy 'from one drug for one target for one disease' to systems pharmacology paradigm including treatment of systems diseases by combination therapy with increasing efficacy [3]. Development of systems biology approaches combined with high-throughput omics technologies revealed mechanisms of a complex action of drugs due to the complexity and cross talk of cellular signalling networks in healthy and pathology. Moreover, it was reported that $22 \%$ of the gene products (enzymes, receptors, ion channels, kinases) are 'druggable' and half of them already are successful targets by approved drugs that ensures a reach database to explore new potential of the approved drugs and their repurpose [3].

Another powerful impulse for drug repurposing was given by new knowledge on disease mechanisms and pathology development. In the case of cancer therapy, adaptation of non-cancer drugs has become possible as a result of acquisition of new knowledge on its connection to inflammation, energy metabolism, lipogenesis, immunity, tumour environment and other cellular processes promoting malignancy. Integrative approach to the investigation of the multiple disease mechanisms was successfully developed in the framework of systems biology that showed its potential in the development of facilitating pathway-focused therapy and discovering of new action modes of approved drugs through their repositioning [6]

Below we discuss systems biology strategies in drug repurposing and highlight three mutually reinforcing approaches which include 1) cellular pathway analysis aiming at the identification of drug effects in downstream and crosstalk pathways relevant to the specific disease; 2) searching for new targets and new mechanisms of action of the repurposed drugs, and 3) bioinformatics approach to comparative pathway-based analyse of microarray data on gene expression response to treatment by repurposed and approved drugs for identification and matching of drug signatures.

We demonstrate these three systems approaches by discussion of different aspects of repurposing of the well-established nonhormonal drugs for treatment osteoporosis, bisphosphonates (BPs) to cancer therapy [7]. BPs such as alendronate, risedronate, zeledronate and others used in clinics abrogate osteoclast-mediated bone resorption by inhibition of key enzymes in the mevalonate pathway, required for synthesis of farnesyl diphosphate (FPP) (Figure 1). FPP is needed for lipid post-translation modification of small GTPase including Ras, Rho, and Rac that ensures their membrane recruitment leading to GTPase co-localisation with their effectors. Inhibition of farnesyl pyrophosphate synthase (FPPS) by BPs prevents the prenylation of GTPase, accumulation of unprenylated proteins in their inactive form

*Corresponding author: Alexey Goltsov, School of Science, Engineering and Technology, Abertay University, Dundee, UK, Tel: +44-1382-308-432; E-mail: a.goltsov@abertay.ac.uk

Rao Papineni Ph.D, Branford, CT, USA. papineni@graduate.hku.hk

Received May 22, 2018; Accepted May 25, 2018; Published June 04, 2018

Citation: Goltsov A, Kowalczyk Z, Papineni RVL (2018) Systems Biology Strategy for Drug Potentiating through Drug Repurposing: Bisphosphonates beyond Osteoporosis Treatment. J Bioanal Biomed 10: e154. doi:10.4172/1948-593X.1000e154

Copyright: @ 2018 Goltsov A, et al. This is an open-access article distributed under the terms of the Creative Commons Attribution License, which permits unrestricted use, distribution, and reproduction in any medium, provided the original author and source are credited. 
Citation: Goltsov A, Kowalczyk Z, Papineni RVL (2018) Systems Biology Strategy for Drug Potentiating through Drug Repurposing: Bisphosphonates beyond Osteoporosis Treatment. J Bioanal Biomed 10: e154. doi:10.4172/1948-593X.1000e154

that induce osteoclast apoptosis [7]. High affinity of BPs to mineral surfaces of bones brings them close to osteoclasts increasing BP uptake that advantages BPs delivery to their target [8].

Increasing evidences of anti-tumour effect of BPs and their effectiveness to inhibit cancer cell adhesion, invasion, proliferation and induce apoptosis have been reported $[8,9]$. To the best of our knowledge, our laboratory showed for the first time binding of osteoporosis drug bisphosphonate to prostate and ovarian cancer tumors non-invasively and its potential drug repurposing candidature (Figure 2) [10]. Mechanism of anticancer activity of BPs can be established through the pathway-based analysis of downstream pathway and cross talk between the BP targeted mevalonate pathway and MAP kinase pathway which is frequently activated in many cancers and is an effective target of numerous anticancer drugs (Figure 1). As discussed above, inhibition of FPPS by BPs prevents the prenylation of GTPase RasGTPase that blocks its recruitment to membrane in vicinity of receptor tyrosine kinase (RTK) and inhibits proliferation signals in MAP kinase pathway in cancer cells [8] (Figure 1).

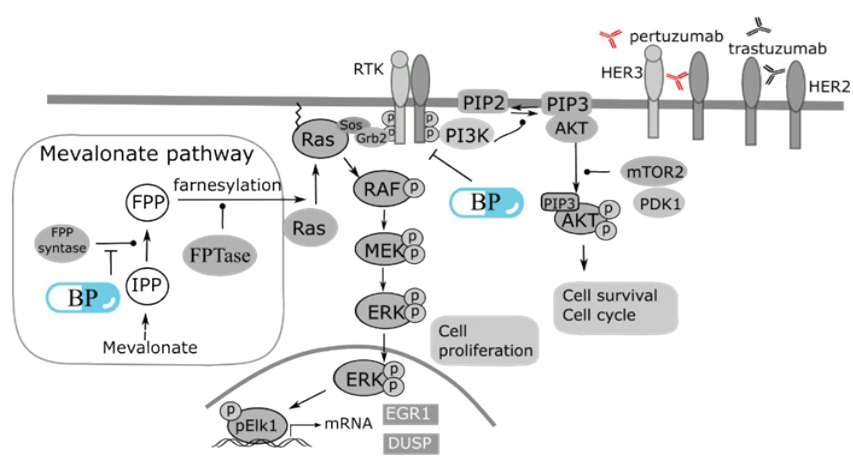

Figure 1: Scheme of molecule targets of BPs in mevalonate and MAP kinase, $\mathrm{PIJK} / \mathrm{AKT}$ pathways in cancer cells. FPTase-farnesyl protein transferase.

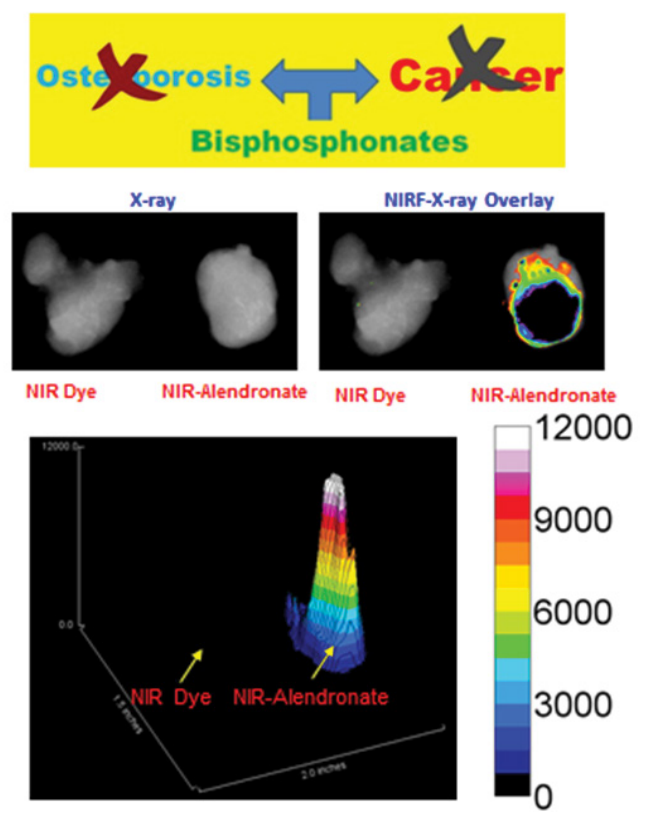

Figure 2: NIR (near-IR) - tricarbocyanine, cyclic enamine dye conjugated alendronate binding to $\mathrm{LNCaP}$ ortho tropic prostate cancer model. (Papineni unpublished observeations).
The second approach of the systems biology strategy to drug repurposing can be demonstrated by identification of the new targets of BPs in RTK signalling pathway relating to cancer progression (Figure 1). As reported, BPs bind to RTK and inhibit downstream cellular signalling in MAP kinase pathways that results in reduction in viability of HER-driver lung and breast cancer cells $[9,11]$. This finding suggests a double action of BPs in cancer cells i.e. a new role as RTK inhibitor is added to its inhibition action relative to RasGTPase activity due to inhibition of its prenylation. Identification of the new BP targets unveiled full therapeutic potential of BPs as a new therapeutics to RTK driven lung, breast, and colon cancers which can be used alone and in combination with chemotherapy and targeted therapy [11].

The third systems approach to drug repurposing mentioned above includes bioinformatic analysis of gene expression profiles to find common genes networks and pathways which are up- and downregulated following the treatment by BPs and established anticancer drugs. Results of this analysis can help to identify new mechanisms of drug action by comparison of cell responses to repurposed drugs with that of drugs with a known mechanism of action. The most interest in this analysis is the genes and pathways contributing to cancer proliferation which are a typical task of the enrichment analysis [12]. In Figure 3 we provided the heatmap for a gene expression change following the treatment by three drugs. The first one is Herceptin, a HER2 inhibitor being an effective drug for HER2 overexpressing breast cancer [13]. The second drug is Pertuzumab which blocks receptor heterodimerization of HER2 and HER3 [14]. The third drug is alendronate, one of commonly used BPs (Figure 3) $[7,11,15,16]$.

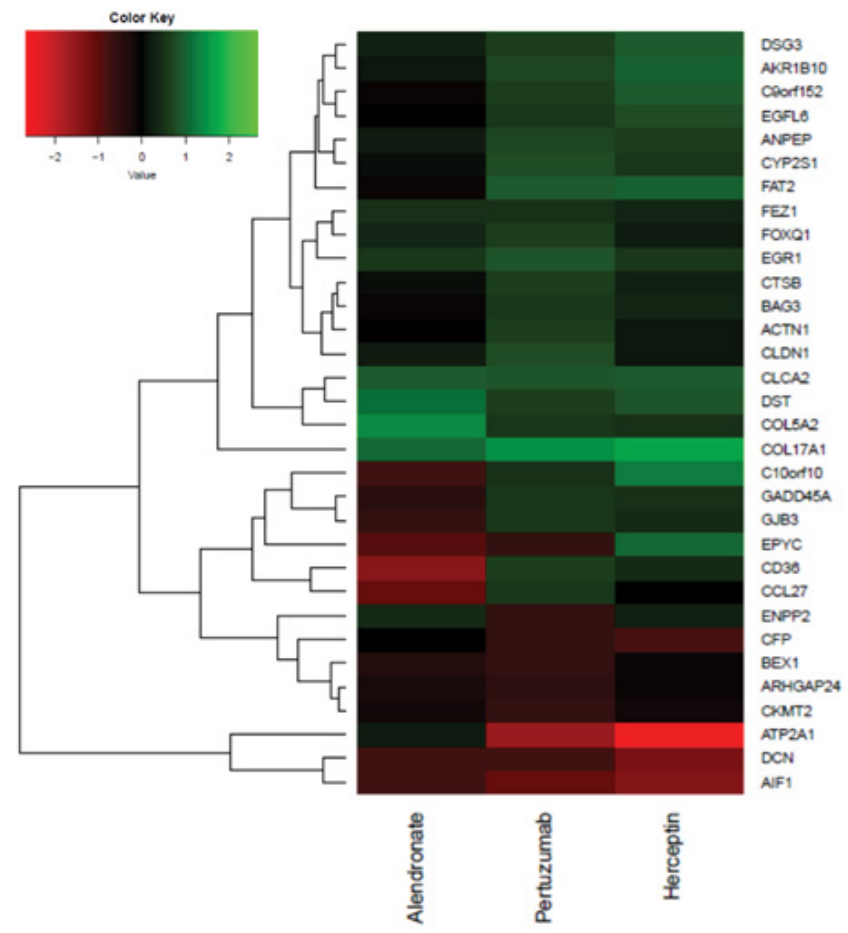

Figure 3: The heatmap representing comparison of the significant gene expression fold change following Pertuzumab, Herceptin treatments of SKOV3 ovarian xenograft tumour $[15,16]$ and alendronate treatment of osteoclasts from peripheral blood mononuclear cells [11]. 
Citation: Goltsov A, Kowalczyk Z, Papineni RVL (2018) Systems Biology Strategy for Drug Potentiating through Drug Repurposing: Bisphosphonates beyond Osteoporosis Treatment. J Bioanal Biomed 10: e154. doi:10.4172/1948-593X.1000e154

Comparison of the gene expression response to these drugs can reveal either similarity or difference in gene profiles. Among common genes, there are genes being under regulation of MAP kinase and PI3K/AKT pathways e.g. EGR1 gene (Early Growth Response 1). Further analysis can be used to confirm that alendronate targets the same pathway in cancer cells as Pertuzumab and Herceptin. Detailed investigation of gene profiles for searching for new targeted pathways can be carried out by using computational tools of enrichment analysis [12] to identify common pathways affected by the anticancer drugs and the repurposed drugs.

Summarising this brief analysis, we advocate systems biology is a powerful approach in supporting and accelerating of drug repurposing and can ensure a potential avenue for the discovery of new modes in an action for the established drugs and help expand their therapeutic potential for the development of a novel therapy.

\section{References}

1. Martelli AM, Buontempo F, McCubrey JA (2018) Drug discovery targeting the mTOR pathway. Clin Sci 132: 543-568.

2. Whitburn J, Edwards CM, Sooriakumaran P (2017) Metformin and prostate cancer: a new role for an old drug. Curr Urol Rep 18: 46.

3. Duran-Frigola M, Mateo L, Aloy P (2017) Drug repositioning beyond the lowhanging fruits. Curr Opin Syst Biol 3: 95-102.

4. Vanhaelen Q, Mamoshina P, Aliper AM, Artemov A, Lezhnina K et al. (2017) Design of efficient computational workflows for in silico drug repurposing. Drug Discov Today 22: 210-222.

5. Loudon JA (2013) Repurposing amlexanox as a run the red light cure-all with read through a no-nonsense approach to personalised medicine. J Bioanal Biomed 5: 079-096.

6. Khalil HS, Mitev V, Vlaykova T, Cavicchi L, Zhelev N (2015) Discovery and development of Seliciclib. How systems biology approaches can lead to better drug performance. J Biotechnol 202: 40-49.

7. Russell RG, Watts NB, Ebetino FH, Rogers MJ (2008) Mechanisms of action of bisphosphonates: similarities and differences and their potential influence on clinical efficacy. Osteoporos Int 19: 733-759.

8. Ottewell PD, Woodward JK, Lefley DV, Evans CA, Coleman RE, et al. (2009) Anticancer mechanisms of doxorubicin and zoledronic acid in breast cancer tumor growth in bone. Mol Cancer Ther 8: 2821-2832.

9. Stachnik A, Yuen T, Iqbal J, Sgobba M, Gupta Y, et al. (2014) Repurposing of bisphosphonates for the prevention and therapy of nonsmall cell lung and breast cancer. Proc Natl Acad Sci U S A 111: 17995-8000.

10. Papineni RV, Udayakumar T, Ahmed M, Pizzonia J, Pollack A (2011) Abstract 5327: In vivo fluorescence imaging of solid tumor-bisphosphonate interaction. Cancer Res 71: 5327-5327.

11. Yuen T, Stachnik A, lqbal J, Sgobba M, Gupta Y, et al (2014) Bisphosphonates inactivate human EGFRs to exert antitumor actions. Proc Natl Acad Sci U S A 111: $17989-17994$

12. Creixell P, Reimand J, Haider S, Wu G, Shibata T, et al. (2015) Pathway and network analysis of cancer genomes. Nat Methods 12: 615-621.

13. Ghosh R, Narasanna A, Wang SE, Liu S, Chakrabarty A, et al (2011) Trastuzumab has preferential activity against breast cancers driven by HER2 homodimers. Cancer Res 71: 1871-1882.

14. Faratian D, Goltsov A, Lebedeva G, Sorokin A, Moodie S, et al. (2009) Systems biology reveals new strategies for personalizing cancer medicine and confirms the role of PTEN in resistance to trastuzumab. Cancer 69: 6713-6720.

15. Sims AH, Zweemer AJ, Nagumo Y, Faratian D, Muir M, et al. (2012) Defining the molecular response to trastuzumab, pertuzumab and combination therapy in ovarian cancer. Br J Cancer 106: 1779-1789.

16. Goltsov A, Deeni Y, Khalil HS, Soininen T, Kyriakidis S, et al. (2014) Systems analysis of drug-induced receptor tyrosine kinase reprogramming following targeted mono- and combination anti-cancer therapy. Cells 3: 563-591. 